\title{
The dynamics of geomagnetic substorms with the WINDMI model
}

\author{
Edmund Spencer ${ }^{1 *}\left(\mathbb{D}\right.$, Sai Krishna Vadepu', Pavithra Srinivas' ${ }^{1}$ Swadesh Patra ${ }^{2}$ and Wendell Horton ${ }^{3}$
}

\begin{abstract}
The global dynamics of substorms are controlled by several key magnetospheric parameters. In this work we obtain quantitative measures of these parameters from a low-order nonlinear model of the nightside magnetosphere called WINDMI. The model uses solar wind and IMF measurements from the ACE spacecraft as input into a system of 8 nonlinear ordinary differential equations. The state variables of the differential equations represent the energy stored in the geomagnetic tail, central plasma sheet, ring current and field-aligned currents. The output from the model is the geomagnetic westward auroral electrojet (AL) index and the Dst index. Intermediate variables of the model are the plasma sheet pressure, geotail current, cross-tail electric field, parallel ion velocity and the pressure gradient current. The values of these variables are controlled by physical parameters of the model, consisting of spatially averaged quantities that are analogous to electric circuit elements. We tune the model to re-produce substorm events, comparing model capability against observations of auroral brightening and the auroral electrojet indices AL from WDC Kyoto and SML from SuperMAG. The model is capable of capturing events within a 10-12-min interval of occurrence, with level of activity comparable to the measured indices.
\end{abstract}

Keywords: WINDMI, Magnetosphere, Substorm

\section{Introduction}

The fundamental processes that result in triggering of a substorm have been a topic of intense debate and research over several decades. The energy variables that strongly influence the buildup and subsequent release of energy in a substorm are the magnetotail lobe current and associated lobe magnetic field, the cross-tail voltage, the plasma sheet magnetic and thermal pressure, and the speed of parallel flows along the stretched tail magnetic field lines. The ionosphere and inner magnetosphere influence the growth phase, onset phase, expansion phase and recovery phase of a typical substorm. These regions may behave very differently under the strongly variable solar wind conditions. Their interaction may be different under various types of substorm activity, for example isolated substorms that occur during relatively quiet periods, storm time substorms or sawteeth events which are

*Correspondence: espencer@southalabama.edu

${ }^{1}$ University of South Alabama, Mobile, USA

Full list of author information is available at the end of the article sometimes referred to as periodic substorms (Partamies et al. 2009).

A standard view of the substorm temporal development is through a nonlinear energy buildup and unloading process (Baker et al. 1999). The growth phase begins when the IMF turns southward for a certain period of time, then plasma sheet thinning develops, and then reconnection occurs. This is followed by a rapid dipolarization during the expansion phase. The growth phase is not always clearly observable because of intermittent northward and southward fluctuations in the IMF. The precise mechanism and sequence of events during substorm onset are still under investigation (Sergeev et al. 2012), but reconnection in the tail is widely accepted to be the point when dipolarization occurs. This begins the expansion phase of a substorm. Finally, after plasma energy is lost through plasma outflow and ohmic dissipation, the substorm moves into the recovery phase and the magnetotail reverts back to a stretched tail configuration.

The nonlinear loading and unloading character of geomagnetic substorms under southward IMF conditions has been investigated by several authors (Klimas et al. 
1992, 1996; Blanchard and McPherron 1993). Vassiliadis et al. (1995) employed a complex nonlinear filter approach for describing the solar wind-magnetosphere coupling and later to predict the AL index (Klimas et al. 1998). Weigel et al. (1999) used a neural network technique to predict the AL index. The nonlinear dynamical WINDMI model was used by Horton et al. (2003) to classify substorms into 3 categories, including the so-called northward turning triggered substorm (Gallardo-Lacourt et al. 2012). Spencer and Patra (2013) investigated the effect of enhanced ionospheric conductance on the substorm dynamics.

Here we proceed to develop and analyze the WINDMI model characteristics following (Horton and Doxas 1996) and (Spencer et al. 2007) as a tool to forecast the loading-unloading type of substorm. We note that a strongly fluctuating solar wind may trigger surges in the $\mathrm{AE}$ index (Pulkkinen et al. 2006), but this effect is not represented in the physics of the WINDMI model. The current version of the WINDMI model is available at the NASA Community Coordinated Modeling Center for real-time forecasts of space weather geomagnetic storm and substorm activity (Mays et al. 2009).

Kalmoni et al. (2015) used auroral brightening signatures from ground-based auroral imagers to identify onset times. These onset times may indicate the beginning of substorms or pseudo-breakup events. Using these onset times as a rough guide, and combining them with AL and SML (SuperMAG lower auroral electrojet index) indices obtained from WDC Kyoto and SuperMAG, respectively, we tune the WINDMI model to produce substorms to establish bounds for the model parameters under different magnetospheric and solar wind conditions.

The paper is outlined as follows. In the next section the WINDMI model is described. In the third section we discuss the data and methodology employed to analyze the substorms. We then present the results of our analysis in the fourth section and finally draw some conclusions in the fifth section.

\section{WINDMI model}

The plasma physics-based nonlinear dynamical WINDMI model uses an equivalent solar wind voltage $V_{\mathrm{sw}}$ generated by solar wind-magnetosphere coupling functions as input to eight ordinary differential equations that accounts for the transfer of power between the global energy components of the nightside magnetosphere. The WINDMI model is discussed in some details in Doxas et al. (2004), Spencer et al. (2007) and more recently in Patra et al. (2011). The equations of the model are given by:

$$
\begin{aligned}
& L \frac{\mathrm{d} I}{\mathrm{~d} t}=V_{\mathrm{sw}}(t)-V+M \frac{\mathrm{d} I_{1}}{\mathrm{~d} t} \\
& C \frac{\mathrm{d} V}{\mathrm{~d} t}=I-I_{1}-I_{\mathrm{ps}}-\Sigma V \\
& \frac{3}{2} \frac{\mathrm{d} p}{\mathrm{~d} t}=\frac{\Sigma V^{2}}{\Omega_{\mathrm{cps}}}-u_{0} p K_{\|}^{1 / 2} \Theta(U)-\frac{p V A_{\mathrm{eff}}}{\Omega_{\mathrm{cps}} B_{\mathrm{tr}} L_{y}}-\frac{3 p}{2 \tau_{E}} \\
& \frac{\mathrm{d} K_{\|}}{\mathrm{d} t}=I_{p s} V-\frac{K_{\|}}{\tau_{\|}} \\
& L_{I} \frac{\mathrm{d} I_{1}}{\mathrm{~d} t}=V-V_{I}+M \frac{\mathrm{d} I}{\mathrm{~d} t} \\
& C_{I} \frac{\mathrm{d} V_{I}}{\mathrm{~d} t}=I_{1}-I_{2}-\Sigma_{I} V_{I} \\
& L_{2} \frac{\mathrm{d} I_{2}}{\mathrm{~d} t}=V_{I}-\left(R_{\mathrm{prc}}+R_{A 2}\right) I_{2} \\
& \frac{\mathrm{d} W_{\mathrm{rc}}}{\mathrm{d} t}=R_{\mathrm{prc}} I_{2}^{2}+\frac{p V A_{\mathrm{eff}}}{B_{\mathrm{tr}} L_{y}}-\frac{W_{\mathrm{rc}}}{\tau_{\mathrm{rc}}}
\end{aligned}
$$

The coupled nonlinear equations track the exchange of electromagnetic, electric and mechanical energy through eight pairs of energy-conserving terms. The remaining terms in each equation describe the loss of energy from the magnetosphere-ionosphere system through plasma outflows, ionospheric losses and ring current particle energy decay.

The coefficients in the model are physical parameters related to the geometrical and plasma configuration of the magnetosphere-ionosphere system. The parameters $L, C, \Sigma, L_{I}, C_{I}$ and $\Sigma_{I}$ are the magnetospheric and ionospheric inductances, capacitances and conductances, respectively. $A_{\text {eff }}$ is an effective aperture for particle injection into the ring current that on the dusk side merges with the Alfven layer Doxas et al. (2004). The resistances in the partial ring current and region- 2 current, $I_{2}$ are $R_{\mathrm{prc}}$ and $R_{A 2}$, respectively, and $L_{2}$ is the inductance of the region- 2 current. The coefficient $u_{0}$ in Eq. 3 is a heat fluxlimiting parameter. 
The energy confinement times for the central plasma sheet, parallel kinetic energy and ring current energy are $\tau_{E}, \tau_{k}$ and $\tau_{\mathrm{rc}}$, respectively. The effective width of the magnetosphere is $L_{y}$, and the transition region magnetic field is given by $B_{t r}$. The pressure gradient current due to the magnetic field curvature is given by $I_{p s}=L_{x}\left(p / \mu_{0}\right)^{1 / 2}$, where $L_{x}$ is the effective length of the earth's magnetotail. The pressure gradient current flows in the y direction to balance the pressure gradient in the $\mathrm{x}$ direction antisunward as given by the equilibrium MHD momentum equation $-\nabla p+\mathbf{J} \times \mathbf{B}=0$. The outputs of the model are the AL and Dst indices, which are taken to be proportional to the magnetospheric field-aligned currents. Estimates of the physical parameters are listed in Spencer et al. (2007). The AL index is calculated from the region 1 current $I_{1}$ index by assuming it to be proportional with a constant $\lambda_{A L}[A / n T]$, resulting in $\Delta B_{A L}=-I_{1} / \lambda_{A L}$.

The input coupling function used for the model is the rectified $v B_{s}$ formula (Reiff and Luhmann 1986), given by:

$$
V_{s w}^{B s}=40(k V)+v_{s w} B_{s}^{I M F} L_{y}^{e f f}(k V)
$$

where $v_{s w}$ is the $\mathrm{x}$-directed component of the solar wind velocity in GSM coordinates, $B_{s}^{I M F}$ is the southward IMF component, and $L_{y}^{e f f}$ is an effective cross-tail width over which the voltage is produced. For northward or zero $B_{s}^{I M F}$, a base viscous voltage value of $4 \mathrm{kV}$ is used as input into the system.

Substorm onsets are triggered in the WINDMI model through a switching function $\Theta(U)$. The pressure unloading function $\Theta(U)=\frac{1}{2}[1+\tanh U]$ where $U=\left(I-I_{c}\right) / \Delta I$ in Eq. (3) is turned on by a geotail critical current $I_{c}$ over an interval $\Delta I$ when a transition to plasma loss along newly opened magnetic field lines with an equivalent parallel thermal flux $q_{\|}$occurs. The parallel thermal flux goes from zero to one as the geotail current $I$ rises to $I_{c}$. The unloading function used here corresponds to current gradient-driven tearing modes or cross-field current instabilities, as outlined in Yoon et al. (2002).

In Fig. 1, we show the result of driving the WINDMI model with the solar wind velocity Vx being $450 \mathrm{~km} / \mathrm{s}$ and IMF Bz of $-6.67 \mathrm{nT}$. This is a synthetic signal which was chosen to make the product of $\mathrm{Vx}$ and $\mathrm{Bz}$ a certain value. With the effective width of the magnetosphere taken to be 10 earth radii, this results in a solar wind voltage of $191 \mathrm{kV}$. A substorm is triggered when $C=1000 F$, $\Sigma=10 S, I_{c}=3.2 M A$ and $\Omega=4000 R_{E}^{3}$.

\section{Substorm data and methodology}

Kalmoni et al. (2015) used a set of substorm and pseudobreakup events to study how the growth rate of auroral beads is related to possible instability mechanisms in the near-earth plasma sheet. We used the same set of events but studied the substorm energy and triggering conditions using solar wind and IMF as drivers. The reason we used the events from that paper is because it would be possible to compare the auroral brightening times to the surges in the westward auroral electrojet due to the aforementioned solar wind driving. There were 17 events in total. Acceptable solar wind data from ACE were available for 13 events, and the model was able to trigger a substorm on 9 events. Of these, only 7 events had all the characteristics of a substorm needed for matching to the Kyoto lower auroral electrojet index AL and the SuperMAG lower auroral electrojet index SML. Four events did not have acceptable ACE data, meaning some data were missing, or unusable. For another 4 events, the solar wind input was not strong enough or did not last long enough for this model to trigger a substorm. Finally, in another 2 substorms, the AL and SML signatures were not present or did not coincide in timing, with the solar wind forcing, propagated to the nose of the magnetosphere.

Several motivating factors were considered when selecting parameters to tune in order to establish the substorm-related characteristics of the model. Firstly, depending on solar wind conditions and auroral electrojet intensities, by fixing the average $B_{z}$ in the central plasma sheet, the effective width $L_{y}$ and $\Omega_{c p s}$, we may estimate the mass density present in the central plasma sheet from the capacitance values needed to trigger a substorm. The plasma sheet $\mathrm{Bz}$ is used implicitly in the calculation of the plasma sheet capacitance $\mathrm{C}$.

Secondly, the perpendicular $v_{E}=\mathbf{E} \times \mathbf{B}$ flow velocity with $B_{z}=10 \mathrm{nT}$ goes as $100 \mathrm{~km} / \mathrm{s}$ per $1 \mathrm{mV} / \mathrm{m}$ electric field. Thus we will be able to track the cross-tail electric field if consistent satellite data could be found in future to constrain the model. Thirdly, from the geotail current we could track how the magnetic curvature $\mathrm{db} / \mathrm{d} s$ changes during the substorm development, which is a condition for ballooning instability, Wong et al. (2001). Fourthly we could use the critical current parameter $I_{c}$ to estimate the conditions when current-driven instabilities may be triggered. Finally, the pressure gradient current $I_{p s}$ in the model is an estimate of the earthward pressure gradient $\mathrm{d} p / \mathrm{d} x$, which is also related to conditions for ballooning instability.

The parameters that have the strongest influence on the strength and character of substorms in the model are the geotail inductance $L$, the plasma sheet capacitance $C$, the critical current $I_{c}$, the heat flux parameter $u_{0}$ and the plasma sheet volume $\Omega_{c p s}$. These parameters are varied in the model in order to capture substorm events. However, in order to minimize the number of independent degrees of freedom, we fixed $\Omega=10000 R_{E}^{3}$ in this work. In addition, we do not show the value of $\Sigma_{I}$, the ionosphere conductivity value, used to scale the output 

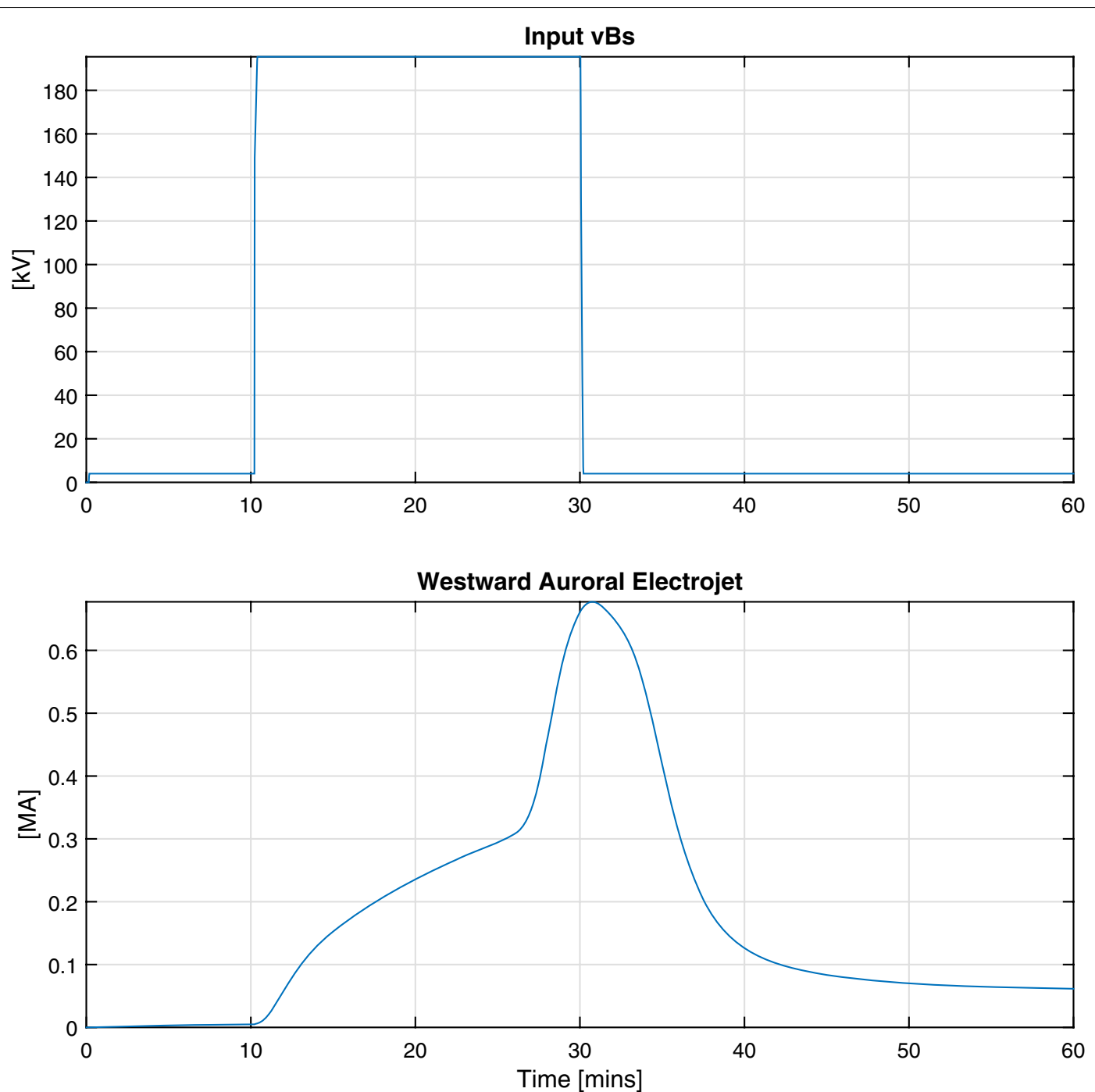

Fig. 1 WINDMI under 191-kV input. The WINDMI model is driven with a synthetic southward IMF voltage signal with the solar wind velocity $V x$ being $450 \mathrm{~km} / \mathrm{s}$ and IMF Bz of $-6.67 \mathrm{nT}$. A substorm is produced when sufficient energy is stored in the magnetotail and a critical current $I_{c}$ is reached

auroral electrojet to the measured AL and SML indices. This scaling is influenced by several factors, including the width and height of the electrojet.

Our analysis consists of two steps. In the first step, we obtain parameters for each of the substorm or pseudobreakup cases in Table 1, by tuning the WINDMI model to produce a substorm driven by solar wind input, coinciding as closely as possible with the auroral brightening times observed by Kalmoni et al. (2015). In the second step, we obtain parameters for each of the cases by tuning the model to match the AL and SML indices as closely as possible. The results are discussed in the next section.
Table 1 WINDMI model parameters obtained when tuned to auroral brightening observations

\begin{tabular}{llllr}
\hline Date & Onset (Mdl. Onset) $(\boldsymbol{\Delta} \boldsymbol{t})$ & $\boldsymbol{\Sigma}(\mathbf{S})$ & $\boldsymbol{I}_{\boldsymbol{C}}$ (MA) & $\boldsymbol{C}(\mathbf{F})$ \\
\hline 22/02/2006 & 06:26 (06:36) (+ 10) & 10 & 3.7 & 5000 \\
07/03/2007 & 05:50 (05:47) (-3) & 10 & 3.4 & 5000 \\
02/10/2008 & $04: 29(04: 23)(-6)$ & 10 & 4.9 & 10000 \\
03/01/2009 & $04: 36(04: 24)(-12)$ & 5 & 3.5 & 7000 \\
24/02/2009 & $07: 32(07: 26)(-5)$ & 5 & 2.5 & 8000 \\
15/03/2009 & $04: 28(04: 24)(-4)$ & 10 & 3.7 & 5000 \\
07/03/2010 & $05: 15(05: 25)(+10)$ & 10 & 3.7 & 5000 \\
\hline
\end{tabular}




\section{Results}

The first set of results obtained were through tuning the model parameters in order that a substorm is triggered around the time of the observation of auroral brightening, and so that a classical growth, expansion and recovery phase can be observed, as shown in Fig. 1. Because the model is driven by solar wind conditions measured at ACE and propagated to the nose of the earth's magnetosphere, the model can only produce a substorm if the solar wind energy is present and sufficient for triggering to occur. In Table 1, we list the events, the associated times when auroral brightening was observed, the onset triggered in the WINDMI model, the time difference between the two and the values of $\Sigma, I_{c}$ and $C$ that were obtained. Overall, the parameters do not vary over large ranges, except that the model onset times are distributed over a 10-12-min interval around the observed auroral brightening times. There were sometimes several substorms triggered in the model due to solar wind forcing, but we chose the start of the event nearest to the observed auroral signatures.

When the model is tuned to the same set of events but now compared to auroral electrojet activity measured by either the AL or SML index, the results are different. The new parameter values obtained in this case are shown in Table 2. Two of the seventeen events prove to be exceptions. For the 2 October 2008 event, no SML or AL signature appears although solar wind forcing is present and the model can be triggered. For the 7 March 2010 event, SML and AL show substorm activity 15-20 min later, but the model triggers too early and cannot be tuned to fit the AL/SML indices. The remaining events have new parameter values. Here we show 3 of these events in Figs. 2, 3 and 4 . In each of these figures, the top panel shows the solar wind-rectified voltage given in Eq. 9, and the bottom panel shows the output of the model compared to the AL and SML indices. We show these events in order to make some observations that may be of interest.

Table 2 WINDMI model parameters obtained when tuned to auroral electrojet activity given by AL/SML indices

\begin{tabular}{lllll}
\hline Date & $\boldsymbol{\Sigma}(\mathbf{S})$ & Ic (MA) & C (F) & $\boldsymbol{\Sigma}_{\boldsymbol{l}}(\mathbf{S})$ \\
\hline 22/02/2006 & 10 & 3.7 & 5000 & 3 \\
$07 / 03 / 2007$ & 9 & 4 & 9000 & 9 \\
$02 / 10 / 2008$ & N/A & N/A & N/A & N/A \\
03/01/2009 & 6 & 4.2 & 8000 & 5 \\
24/02/2009 & 2 & 3.5 & 15000 & 4 \\
15/03/2009 & 5 & 4 & 20000 & 5 \\
$07 / 03 / 2010$ & N/A & N/A & N/A & N/A \\
\hline
\end{tabular}

For the event on 22 February 2006, the auroral observations of brightening put the onset at roughly 6:26 UT. From Fig. 2, we observe that a burst of activity occurs before the event, and the WINDMI model triggers twice, once before the brightening, and once $10 \mathrm{~min}$ after. Because the model nonlinear behavior correlates strongly with the loading-unloading paradigm of substorms, the solar wind input provides energy that needs time to accumulate before unloading can be triggered.

For the event on 07 March 2007, we make two observations, referring to Fig. 3. First, there is sufficient energy in the solar wind to cause the model to produce strong substorm activity before the auroral brightening observations, but the AL and SML indices show no activity. Second, the model output corresponds well with the AL and SML indices after the brightening occurs, building up to a substorm and subsequently recovering. We observe also that the SML index shows a sharp spike toward the end of the substorm period, but this is not seen in the AL index.

For the event on 07 March 2010, shown in Fig. 4, the solar wind input decays early, but the AL and SML indices builds up into what looks like a large substorm. The same signatures appeared on 02 October 2008. For these two events, we were unable to trigger what appears to be a longer substorm that occurred over roughly 70-80 min, according to the AL and SML signatures. Whether this is an internally triggered mode is unclear.

The differences between the AL and SML are perhaps due to the way the indices are calculated from station observations. There are three possible reasons: (1) differences in baseline technique, (2) Kyoto has stations that are not yet included in SuperMAG, (3) errors in data. However, this requires further investigation.

\section{Conclusions}

In this work we have used the WINDMI model to study substorms from the point of auroral brightening signatures as well as the AL and SML indices. The WINDMI model can be used to identify substorm triggering, based on input solar wind conditions, for a subset of cases when there is sufficient energy in the solar wind to drive events. We used the onset times identified from auroral brightening and then compared the WINDMI triggering and subsequent behavior to AL and SML activity. However, it appears that some substorms cannot be explained with this type of loading-unloading model.

The WINDMI model is a fast near real-time model running at the NASA Community Coordinated Modeling Center (CCMC), giving forecasts of substorms and storms from solar wind data obtained from satellites at the L1 point (ACE or DSCOVR). The motivation for our study is firstly to improve the forecasting capability of the 


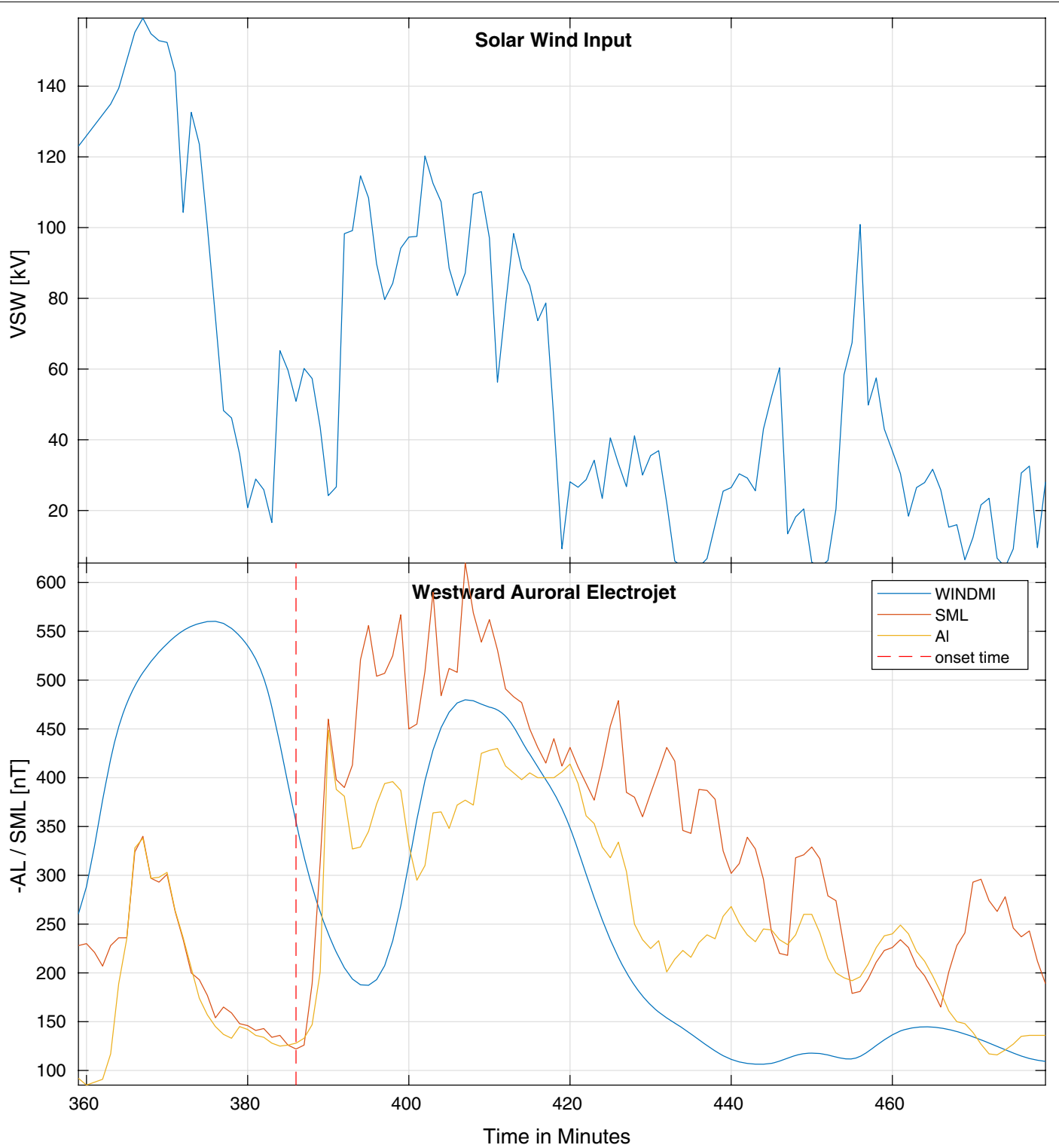

Fig. 2 Event 06:26 UT 22 February 2006: In the bottom panel, substorm onset time from auroral observations is depicted with the red-dotted line. The WINDMI model output is compared to the AL and SML indices. The top panel shows the solar wind input measured by the ACE satellite and propagated to the nose of the magnetosphere

model and secondly to track intermediate variables in the model and use them as a proxy to compare to satellite data. The parameter and state variable values in the model could be used to establish bounds for instabilities that trigger substorm onset. However, many issues remain to be resolved. The model can capture a subset of 


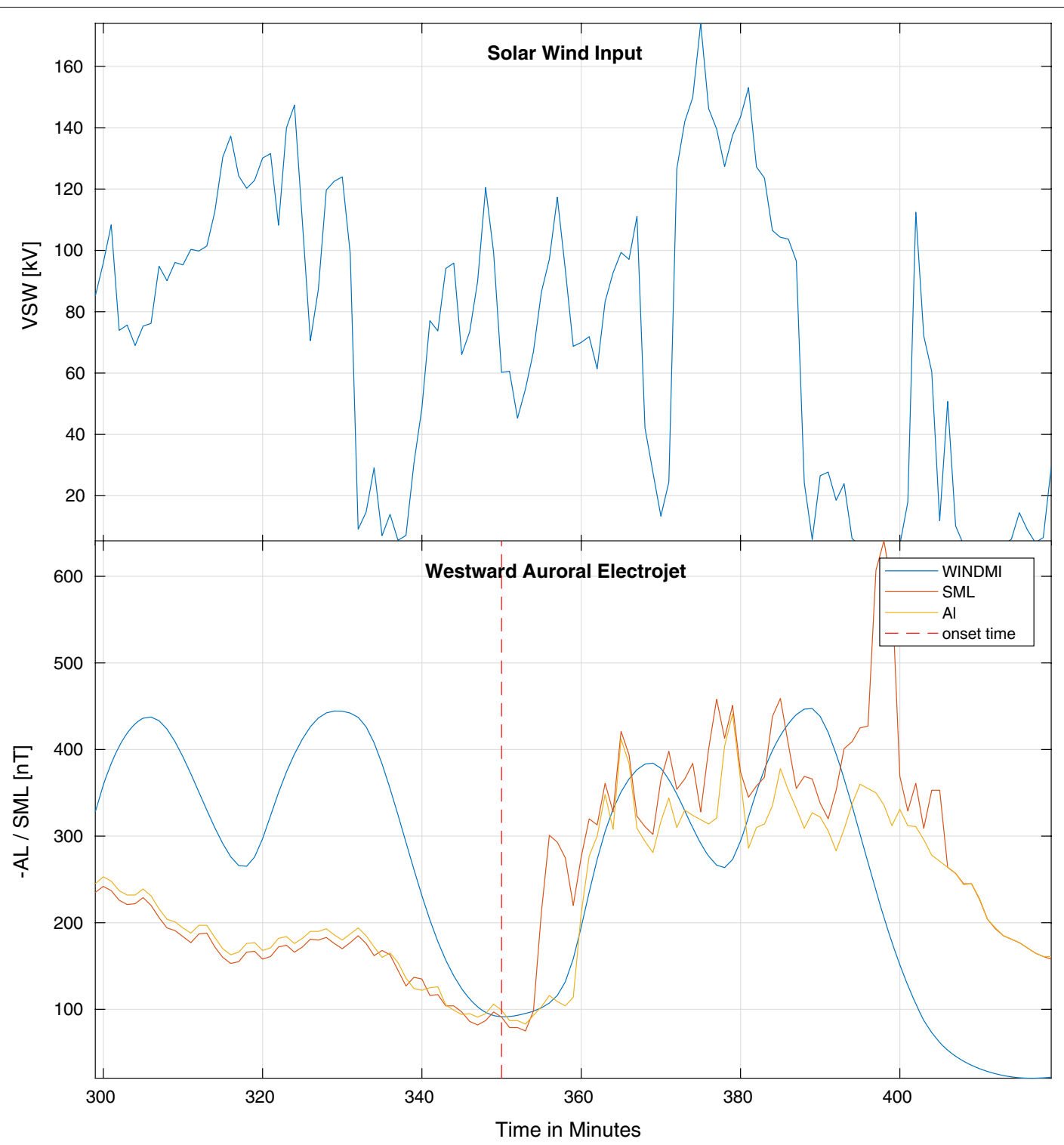

Fig. 3 Event 05:50 UT 07 March 2007: In the bottom panel, substorm onset time from auroral observations is depicted with the red-dotted line. The WINDMI model output is compared to the AL and SML indices. The top panel shows the solar wind input measured by the ACE satellite and propagated to the nose of the magnetosphere

events, but sometimes overpredicts the occurrence (multiple triggers) or intensity of an event, and sometimes, it does not capture an event, especially when the solar wind parameters are not consistent with the AL and SML indices. Additionally, we need measurements of solar wind parameters, ground-based indices, satellite measurements and identification of substorm signatures to all coincide. There are many instances when one or more do 


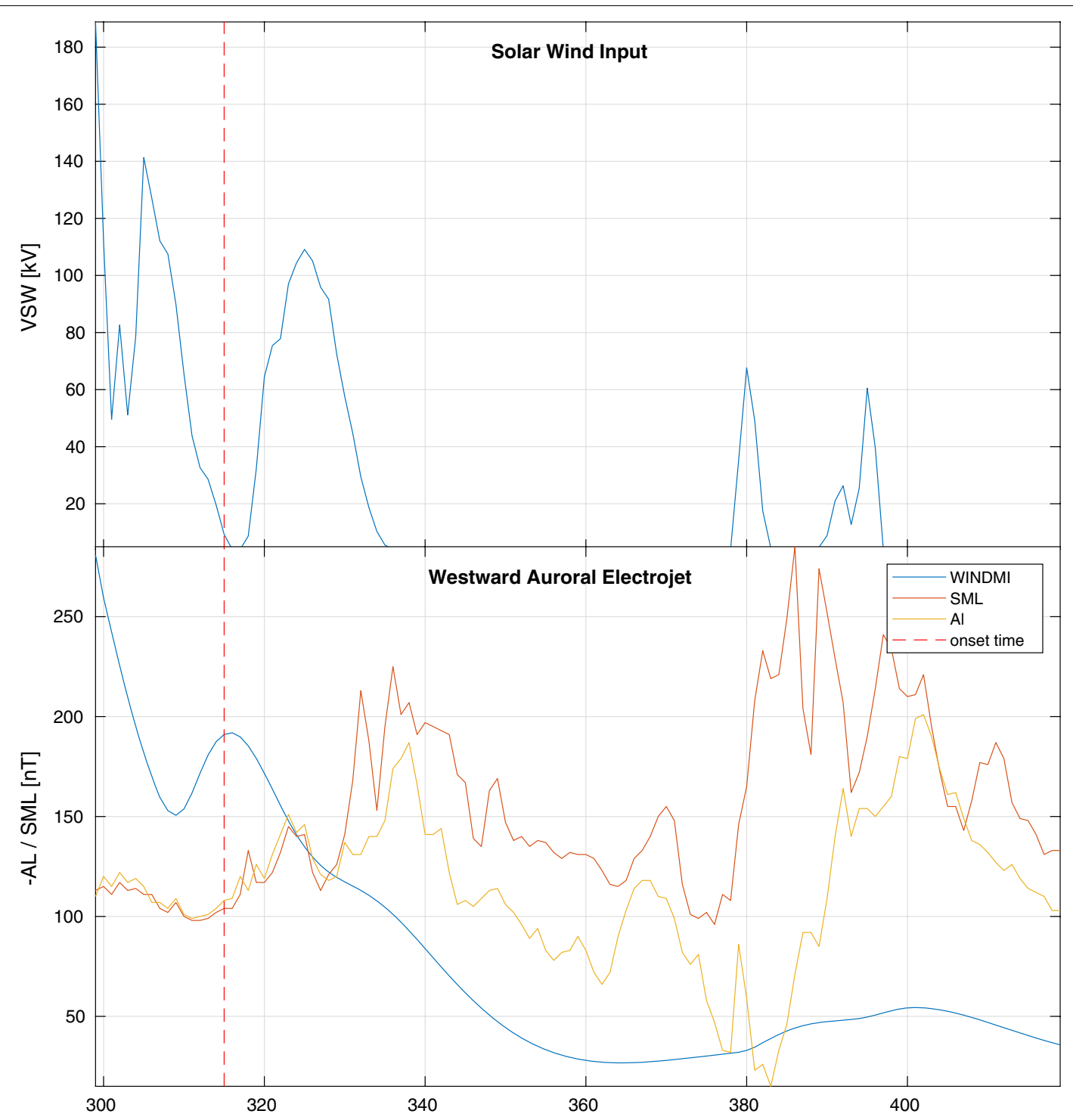

Fig. 4 Event 05:15 UT 07 March 2010: In the bottom panel, substorm onset time from auroral observations is depicted with the red-dotted line. The WINDMI model output is compared to the AL and SML indices. The top panel shows the solar wind input measured by the ACE satellite and propagated to the nose of the magnetosphere

not exist, or are not easily interpretable, to help constrain the model. Investigation of these issues is left for future work.

\section{Authors' contributions}

ES conceived of the study, performed the initial data analysis, model preparation and drafted the manuscript. SKV and PS performed further data analysis and model tuning. SP contributed to the interpretation of the results. WH provided the theoretical values and acceptable ranges for the WINDMI model parameters. All authors read and approved the final manuscript.

\section{Author details}

${ }^{1}$ University of South Alabama, Mobile, USA. ${ }^{2}$ University of Oslo, Oslo, Norway.

${ }^{3}$ University of Texas, Austin, USA.

\section{Acknowledgements}

The authors acknowledge following data providers: NASA SPDF for the ACE satellite data, WDC Kyoto for the AL indices, SuperMAG for the SML indices. This work is partially supported by NSF Grant 1655280.

\section{Competing interests}

The authors declare that they have no competing interests. 


\section{Availability of data and materials}

All spacecraft and ground-based data are publicly available. The WINDM model is available to run on request at NASA CCMC, or alternatively as a request to the authors.

\section{Funding}

This material is based upon work supported by the NSF EPSCoR RII-Track-1 Cooperative Agreement OIA-1655280.

\section{Publisher's Note}

Springer Nature remains neutral with regard to jurisdictional claims in published maps and institutional affiliations.

Received: 21 February 2018 Accepted: 25 June 2018

Published online: 11 July 2018

\section{References}

Baker D, Pulkkinen T, Buchner J, Klimas A (1999) Substorms: a global instability of the magnetosphere-ionosphere system. J Geophys Res 104(A7):14,601-14,611

Blanchard G, McPherron R (1993) A bimodal representation of the response function relating the solar wind electric field to the al index. J Adv Space Res 13(71):71-74

Doxas I, Horton W, Lin W, Seibert S, Mithaiwala M (2004) A dynamical model for the coupled inner magnetosphere and tail. IEEE Trans Plasma Sc 32(4):1443-1448

Gallardo-Lacourt B, Nishimura Y, Lyons L, Donovan E (2012) External triggering of substorms identified using modern optical versus geosynchronous particle data. Ann Geophys 30:667-673

Horton W, Doxas I (1996) A low-dimensional energy-conserving state space model for substorm dynamics. J Geophys Res 101(A12):27,223-27,237

Horton W, Weigel RS, Vassiliadis D, Doxas I (2003) Substorm classification with the windmi model. Nonlinear Processes Geophys 10:363-371

Kalmoni NME, Rae IJ, Watt CEJ, Murphy KR, Forsyth C, Owen CJ (2015) Statistical characterization of the growth and spatial scales of the substorm onset arc. J Geophys Res 120(10):8503-8516. https://doi. org/10.1002/2015JA021470

Klimas A, Baker D, Vassiliadis D, Roberts D, Fairfield D, Buchner J (1992) A nonlinear analog model of geomagnetic activity. J Geophys Res 97:12253-12266

Klimas A, Vassiliadis D, Baker D, Roberts D (1996) The organized nonlinear dynamics of the magnetosphere. J Geophys Res 101:13089-13113
Klimas A, Valdivia J, Vassiliadis D, Baker D (1998) Al index prediction using dataderived nonlinear prediction filters. In: Chang T, Jasperse JR (eds) Physics of space plasmas. MIT Center for Theoretical Geo/Cosmo Plasma Physics, Cambridge

Mays ML, Horton W, Spencer E, Kozyra J (2009) Real-time predictions of geomagnetic storms and substorms: use of the solar wind magnetosphereionosphere system model. Space Weather. https://doi.org/10.1029/2008S W000459

Partamies N, Pulkkinen T, McPherron R, McWilliams K, Bryant C, Tanskanen E, Singer H, Reeves G, Thomsen M (2009) Statistical survey on sawtooth events, smcs and isolated substorms. J Adv Space Res 44:376-384

Patra S, Spencer E, Horton W, Sojka J (2011) Study of Dst/ring current recovery times using the WINDMI model. J Geophys Res. https://doi. org/10.1029/2010JA015824

Pulkkinen A, Klimas A, Vassiliadis D, Uritsky V (2006) Role of stochastic fluctuations in the magnetosphere-ionosphere system: a stochastic model for the AE index variations. J Geophys Res. https://doi.org/10.1029/2006J A011661

Reiff PH, Luhmann JG (1986) Solar wind control of the polar-cap voltage. In: Kamide Y, Slavin JA (eds) Solar wind-magnetosphere coupling. Terra Sci, Tokyo, pp 453-476

Sergeev VA, Angelopoulos V, Nakamura R (2012) Recent advances in understanding substorm dynamics. Geophys Res Lett. https://doi. org/10.1029/2012GL050859

Spencer E, Patra S (2013) The effect of nonlinear ionospheric conductivity enhancement on magnetospheric substorms. Nonlinear Process Geophys 20(3):429-435. https://www.nonlin-processes-geoph ys.net/20/429/2013/

Spencer E, Horton W, Mays ML, Doxas I, Kozyra J (2007) Analysis of the 3-7 October 2000 and 15-24 April 2002 geomagnetic storms with an optimized nonlinear dynamical model. J Geophys Res. https://doi. org/10.1029/2006JA012019

Vassiliadis D, Klimas A, Baker D, Roberts D (1995) A description of solar-wind magnetosphere coupling based on nonlinear filters. J Geophys Res 13(71):3495-3512

Weigel R, Horton W, Tajima T, Detman T (1999) Forecasting auroral electrojet activity from solar wind input with neural networks. Geophys Res Lett 26(10):1353-1356

Wong $\mathrm{H}$, Horton W, Dam JV, Crabtree C (2001) Low frequency stability of geotail plasma. Phys Plasmas 8:2415-2424

Yoon P, Lui A, Sitnov M (2002) Generalized lower-hybrid drift instabilities in current sheet equilibrium. Phys Plasma 9(5):1526-1538

\section{Submit your manuscript to a SpringerOpen ${ }^{\circ}$ journal and benefit from:}

- Convenient online submission

- Rigorous peer review

- Open access: articles freely available online

- High visibility within the field

- Retaining the copyright to your article

Submit your next manuscript at springeropen.com 